\title{
DESEMPENHO DE CULTIVARES DE SOJA DE DIFERENTES CICLOS EM SEMEADURAS DE DEZEMBRO, NA REGIÃO DO PLANALTO MÉDIO DO RIO GRANDE DO SUL
}

\section{PERFORMANCE OF DIFFERENT CYCLE SOYBEAN CULTIVARS IN DECEMBER SEEDINGS IN THE “PLANALTO MÉDIO” OF RIO GRANDE DO SUL, BRAZIL}

\section{Emídio Rizzo Bonato $^{1}$ Cláudia Erna Lange ${ }^{2}$ Paulo Fernando Bertagnolli $^{3}$}

\section{RESUMO}

Com o objetivo de avaliar o comportamento de cultivares de soja de diferentes ciclos, em semeaduras realizadas entre os dias 10 e 30 de dezembro, na região do Planalto Médio do Rio Grande do Sul, foram conduzidos em Cruz Alta, nos anos agrícolas de 1994/95 a 1997/98, e em Passo Fundo, nos anos de 1994/95 e 1997/98, ensaios com cinco cultivares de soja de ciclo precoce, oito de ciclo médio e cinco de ciclos semitardio e tardio. Os ensaios foram organizados em blocos ao acaso, com três repetições. Para evitar a interferência de plantas de cultivares de ciclos diferentes sobre as das parcelas vizinhas, essas foram separadas em 1,0m, além de eliminar-se uma fileira de cada lado de cada parcela. A análise de variância conjunta para rendimento de grãos foi efetuada desdobrando-se os efeitos das cultivares em cultivares de ciclos precoce, médio, semitardio/tardio e entre ciclos. $O$ mesmo procedimento foi adotado com a interação cultivares $x$ ambientes. Foram constatadas diferenças altamente significativas entre ambientes $e$ entre cultivares. Entretanto, não foram constatadas diferenças significativas entre os ciclos das cultivares e entre a interação cultivares $x$ ambientes. Igualmente, não foram verificadas limitações quanto à arquitetura de plantas. Os resultados mostram que as atuais cultivares de soja, independentemente de ciclo, apresentam potencial produtivo semelhante, em semeaduras realizadas no período de 10 a 30 de dezembro.

Palavras-chave: Glycine max, época de semeadura, cultivares. SUMMARY

An experiment using five early cycle, eight medium cycle, and five semilate and late cycle soybean cultivars was conducted in Cruz Alta, in the growing seasons of 1994/95 up to

\begin{abstract}
1997/98, and Passo Fundo, in 1994/95 and 1997/98, Planalto Médio region of the State of Rio Grande do Sul, Brazil, to evaluate the behavior in seedings made between 10 and 30 December. An experimental design of complete randomized blocks with three replications was used. To avoid interference by plants of different cultivars, neighboring plots were kept $1.0 \mathrm{~m}$ apart and also one row in each side of the plots was eliminated prior to harvest. Joint variation analysis for yield was made by splitting the cultivars in early cycle, medium cycle, semilate and late cycle cultivars, and between cycles. The same procedure was followed for the cultivar $x$ environments interaction. Highly significant differences were obtained between environments and among cultivars. Nevertheless, no significant differences were found between cultivar cycles nor between cultivars $x$ environments interaction. Likewise, no limitations related to plant architecture were observed. The results show that the soybean cultivars available currently have similar yield potential when sowed from December 10 to December 30, regardless of their cycle.
\end{abstract}

Key words: Glycine max, seeding date, cultivars.

\section{INTRODUÇÃO}

A pesquisa recomenda para a região do Planalto Médio do Rio Grande do Sul, o uso de cultivares de soja de ciclos semitardio e tardio para as semeaduras realizadas após o mês de novembro (REUNIÃO..., 1999). Essa recomendação baseia-se em estudos desenvolvidos na década de 70 e início

\footnotetext{
${ }^{1}$ Engenheiro Agrônomo, Doutor, Embrapa Trigo, CP 451, 99001-970, Passo Fundo, RS. Bolsista do CNPq. E-mail: bonato@cnpt.embrapa.br. Autor para correspondência.

${ }^{2}$ Engenheiro Agrônomo, Mestre, Fundacep, aluno do Programa de Pós-graduação em Fitotecnia, Universidade Federal do Rio Grande do Sul. E-mail: mtelli@pro.via-rs.com.br.

${ }^{3}$ Engenheiro Agrônomo, Doutor, Embrapa Trigo, CP 451, 99001-970 Passo Fundo, RS. E-mail: bertag@ cnpt.embrapa.br. Recebido para publicação em 06.11.00. Aprovado em 28.03.01
} 
dos anos 80, os quais levaram em consideração as condições de temperatura, de umidade, de fotoperíodo e os seus efeitos sobre o crescimento e desenvolvimento de soja em semeadura tardia (BERLATO \& WESTPHALEN, 1971; MOTA $\boldsymbol{e t}$ al.,1973; BARNI et al., 1978; BARNI \& BERGAMASCHI, 1981: BARNI et al., 1985).

A limitação que as cultivares de soja de ciclos precoce e médio disponíveis, na época da realização desses estudos, apresentavam para serem usadas em semeaduras de dezembro era a sensível diminuição de porte e, conseqüentemente, a diminuição de rendimento de grãos, como resultado da redução do tempo para o florescimento, decorrente do encurtamento do fotoperíodo a partir de 22 de dezembro (MOTA et al., 1973; BARNI \& BERGAMASCHI, 1981: BARNI et al., 1985).

A necessidade de semear soja até meados de dezembro no Rio Grande do Sul é uma realidade ditada pela deficiência hídrica, que normalmente ocorre em novembro, e pela colheita tardia das culturas de inverno que antecedem à de soja.

BONATO et al. (1998) sugeriram que as cultivares de soja de ciclos precoce e médio, lançadas mais recentemente, não apresentam limitação quanto à altura de planta e à inserção de legumes inferiores em semeaduras realizadas até meados de dezembro. Em razão disso, o objetivo deste estudo foi avaliar o comportamento de cultivares de soja de diferentes ciclos, em semeaduras realizadas entre 10 a 30 de dezembro, na região do Planalto Médio do Rio Grande do Sul.

\section{MATERIAL E MÉTODOS}

Foram avaliadas cinco cultivares de soja de ciclo precoce (Cep 16-Timbó, FT-Saray, IAS 5, Ivorá e Ocepar 14), oito de ciclo médio (Bragg, BR4, BR-16, Cep 12-Cambará, Davis, IAS 4, Ipagro 21 e RS 7-Jacuí) e cinco de ciclos semitardio e tardio (Cep 20-Guajuvira, FT-Abyara, RS 9-Itaúba, Cobb e RS 5-Esmeralda), que estiveram entre as indicadas para cultivo no Rio Grande do Sul no período de 1994/95 a 1997/98. O trabalho foi conduzido em Cruz Alta, nos anos agrícolas de 1994/95 a 1997/98, e em Passo Fundo, nos anos de 1994/95 e 1997/98, constituindo seis ambientes. A semeadura em todos os anos e nos dois locais foi realizada em datas compreendidas no período de 10 a 30 de dezembro, após a época de semeadura recomendada pela pesquisa (REUNIÃO..., 1994).

Os ensaios foram organizados segundo o delineamento experimental de blocos ao acaso, com três repetições. As parcelas tinham área total de $2,0 \mathrm{~m} \times 5,0 \mathrm{~m}$, e útil de $1,0 \mathrm{~m} \times 4,0 \mathrm{~m}$. As fileiras foram espaçadas $0,5 \mathrm{~m}$, contendo 20 sementes viáveis por metro linear, procurando-se obter uma população equivalente a 400.000 plantas por hectare. Para evitar a interferência de plantas de cultivares de ciclos diferentes sobre as das parcelas vizinhas, essas foram separadas em 1,0m, além de eliminar-se uma fileira de cada lado de cada parcela. As informações foram colhidas nas duas fileiras centrais, desconsiderando-se $0,5 \mathrm{~m}$ em cada extremidade.

As adubações foram realizadas de acordo com a análise química do solo, segundo as recomendações técnicas para a cultura na região.

As análises do rendimento de grãos foram feitas com os dados colhidos nos seis ambientes, enquanto nas de alturas de plantas e de inserção dos legumes inferiores não foram considerados os dados de Cruz Alta, 1997/98, por terem sido colhidos somente em uma repetição.

As análises de variância foram feitas desdobrando-se os efeitos das cultivares em cultivares de ciclos precoce, médio e semitardio/tardio e entre ciclos. $\mathrm{O}$ mesmo procedimento foi adotado, nas análises conjuntas, para avaliar a interação cultivares $\mathrm{x}$ ambientes (Tabela 1). As médias foram comparadas pelo teste de Tukey, em nível de 5\% de probabilidade de erro.

\section{RESULTADOS E DISCUSSÃO}

Os seis ensaios apresentaram coeficientes de variação entre $7,9 \%$ e $16,4 \%$, os quais podem se considerados como baixos a médios, segundo LÚCIO et al. (1999), indicando boa confiabilidade dos resultados apresentados. Nos ensaios realizados em Cruz Alta, nas safras de 1994/95 e de 1995/96, não foram constatadas diferenças significativas entre rendimento de grãos das 18 cultivares, em nível de 5 $\%$ de probabilidade de erro. Nos demais ensaios, o teste $\mathrm{F}$ identificou diferenças significativas, em 
Tabela 1 - Análise conjunta da variância do rendimento de grãos em kg/ha obtido no experimento de cultivares de soja de diferentes ciclos em Cruz Altas (RS) nas safras agrícolas de 1994/95 a 1997/98 e em Passo Fundo (RS) nas safras de 1994/95 e de 1997/98. Passo Fundo (RS), 2000.

\begin{tabular}{lcc}
\hline Fonte de variação & GL & QM $^{1}$ \\
\hline Ambientes & 5 & $5.862 .689,5704^{* *}$ \\
Cultivares & 17 & $137.683,1155^{* *}$ \\
Cvs. de ciclo precoce & 4 & $277.289,4167^{* *}$ \\
Cvs. de ciclo médio & 7 & $121.397,0208^{*}$ \\
Cvs. de ciclos semitardio e tardio & 4 & $68.164,8000$ \\
Entre ciclos & 2 & $54.508,4756$ \\
Cultivares x Ambientes & $85(62)$ & $46.519,7076$ \\
Cvs. de ciclo precoce x Ambientes & 20 & $50.600,4567$ \\
Cvs. de ciclo médio x Ambientes & 35 & $43.653,1637$ \\
Cvs. de ciclos semitardio e tardio x Ambientes & 20 & $35.024,4800$ \\
Entre ciclos x Ambientes & 10 & $71.381,5686$ \\
Resíduo médio & $204(139)$ & $95.590,3942$ \\
\hline
\end{tabular}

$1 *$ e $* *$ indicam significância aos níveis de 5 e $1 \%$, respectivamente, segundo o teste de F.

Passo Fundo, nos anos agrícolas de 1994/95 e de 1997/98, e em Cruz Alta, em 1996/97 e em 1997/98.

Com exceção dos ensaios conduzidos em Passo Fundo nos anos de 1994/95 e 1997/98, não foram observadas diferenças significativas no rendimento médio entre ciclos. Em Passo Fundo, no ano de 1994/95, o grupo de cultivares de ciclos semitardio e tardio apresentou rendimento de grãos significativamente inferior ao das cultivares de ciclos precoce e médio. Em 1997/98, o menor rendimento foi observado nas cultivares de ciclo precoce (Tabela 2).

A análise conjunta evidenciou diferenças significativas entre ambientes e entre cultivares. Entre as 18 cultivares avaliadas, Cep 16-Timbó apresentou rendimento médio nos seis ambientes inferior ao da cultivar IAS 4, porém semelhante ao das demais. A análise conjunta das cultivares dentro de cada ciclo mostrou que no grupo das precoces, a cultivar Cep 16-Timbó apresentou rendimento inferior ao das outras quatro cultivares avaliadas. No grupo das de ciclo médio, Bragg teve rendimento inferior à IAS 4, mas semelhante ao das demais. Já no grupo das de ciclos semitardio e tardio, o rendimento não diferiu significativamente entre as cinco cultivares estudadas (Tabela 2).

Não foram verificadas, pela análise conjunta do rendimento de grãos, diferenças significativas entre os ciclos das cultivares, confirmando o que foi constatado em quatro dos seis ambientes estudados. Ausência de significância foi também observada na interação de cultivares $\mathrm{x}$ ambientes e nos desdobramentos dessa interação, incluindo a ciclos $\mathrm{x}$ ambientes (Tabela 1).

Os resultados deste estudo evidenciam claramente que há grande semelhança entre o rendimento de grãos das cultivares de soja indicadas para cultivo no Rio Grande do Sul, quando semeadas a partir do dia 10 de dezembro, mesmo que possuam diferentes ciclos. Isso comprova que as atuais cultivares de soja experimentam, em semeaduras realizadas após a época considerada ideal pela pesquisa, as mesmas condições de expressarem seu potencial produtivo, independentemente de ciclo. As recomendações atuais continuam orientando que as semeaduras realizadas em dezembro devem ser feitas com cultivares de ciclos semitardio e tardio (REUNIÃO...,1999).

O desempenho semelhante de cultivares de soja de ciclos precoce, médio e semitardio/tardio, em semeaduras realizadas em meados de dezembro, foi observado por BONATO et al. (1998). Esse comportamento difere do evidenciado em estudos realizados com as cultivares disponíveis no início da década de 80, quando o menor rendimento de grãos das cultivares de ciclo precoce, em comparação com as de ciclos semitardio e tardio, em semeaduras de dezembro, era determinado pelo baixo porte de plantas e pela baixa inserção de vagens inferiores (BARNI \& BERGAMASCHI, 1981: BARNI et al., 1985).

As cultivares de ciclo curto desenvolvidas a partir do final dos anos 80 possuem desenvolvimento maior do que as usadas anteriormente a esse período. Isso reduz a limitação de seu cultivo em semeaduras tardias, imposta pelo fotoperíodo. RUBIN (1995), avaliando o ganho genético obtido pelo melhoramento genético no Rio Grande do Sul para diversas características de importância agronômica em soja, em quarenta anos, estimou um aumento significativo de $0,77 \mathrm{~cm} /$ ano na estatura das cultivares de ciclo precoce, verificando que, para as de ciclos semitardio e tardio ocorreu um decréscimo da ordem de $0,79 \mathrm{~cm} / \mathrm{ano}$. A estatura da planta das cultivares de ciclo médio, por outro lado, 
Tabela 2 - Resultados médios de rendimento de grãos, em kg/ha, obtidos nos experimentos de cultivares de soja de diferentes ciclos em Cruz Alta (RS) nas safras agrícolas de 1994/95 a 1997/98 e em Passo Fundo (RS) nas safras de 1994/95 e de 1997/98. Passo Fundo (RS), 2000

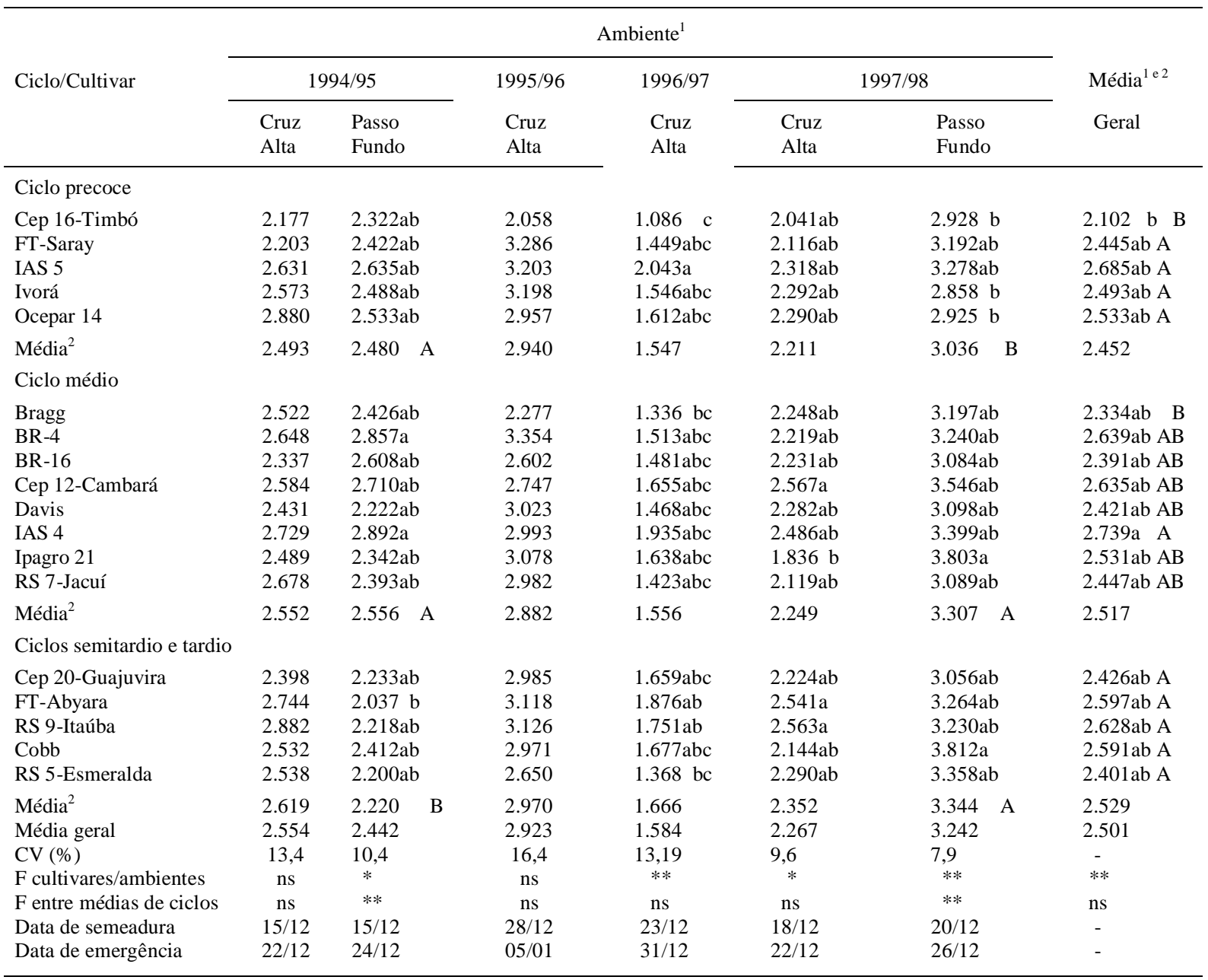

1 Médias não seguidas pela mesma letra minúscula, nas colunas, indicam que as cultivares dentro de cada ambiente e na média geral, independentemente de ciclo, diferem segundo o teste de Tukey, em nível de $5 \%$ de probabilidade de erro.

${ }^{2}$ As médias de cada ciclo ,no mesmo ambiente, e das cultivares, na última coluna, não seguidas pela mesma letra maiúscula, diferem segundo o teste de Tukey, em nível de $5 \%$ de probabilidade de erro.

não foi alterada significativamente nesse período. No presente estudo, constatou-se que a altura de plantas na maturação variou de ano para ano no mesmo local, tendo, em média, apresentado maior porte as plantas das cultivares de ciclos semitardio e tardio, embora não se tenha constatado limitações em nenhum ciclo (Tabela 3). Entre os ambientes estudados, constatou-se que diversas cultivares de todos os ciclos mostraram limitações quanto às alturas de plantas e de inserção dos legumes inferiores, ou seja menores que 60 e $13 \mathrm{~cm}$, respectivamente, apenas em Cruz Alta, em 1995/96, ano em que o ensaio foi instalado muito tardiamente, 23 de dezembro, em virtude da intensa seca registrada na região (Tabelas 3 e 4).

O potencial produtivo das cultivares de soja de todos os ciclos sofre redução em semeaduras tardias. BONATO et al. (1998) mostraram que a redução média do rendimento de grãos ocorrida em semeaduras de meados de dezembro, em comparação com as de meados de novembro, foi semelhantes para as cultivares de todos os ciclos: 17,3\%, nas precoces; $17,1 \%$ nas de ciclo médio; e 19,7\% nas de ciclos semitardio/tardio. Isso explica os rendimentos de grãos 
Tabela 3 - Resultados médios de altura de plantas, em cm, obtidos nos experimentos de cultivares de soja de diferentes ciclos em Cruz Alta (RS) nas safras agrícolas de 1994/95 a 1996/97 e em Passo Fundo (RS) nas safras de 1994/95 e de 1997/98. Passo Fundo (RS), 2000 .

\begin{tabular}{|c|c|c|c|c|c|c|}
\hline \multirow{3}{*}{ Ciclo/Cultivar } & \multicolumn{5}{|c|}{ Ambiente $^{1}$} & \\
\hline & \multicolumn{2}{|c|}{$1994 / 95$} & \multirow{2}{*}{$\begin{array}{l}\text { 1995/96 } \\
\text { Cruz } \\
\text { Alta }\end{array}$} & \multirow{2}{*}{$\begin{array}{l}\text { 1996/97 } \\
\text { Cruz } \\
\text { Alta }\end{array}$} & \multirow{2}{*}{$\begin{array}{l}\text { 1997/98 } \\
\text { Passo } \\
\text { Fundo }\end{array}$} & \multirow{2}{*}{$\begin{array}{l}\text { Média }^{1 \text { e } 2} \\
\text { Geral }\end{array}$} \\
\hline & $\begin{array}{l}\text { Cruz } \\
\text { Alta }\end{array}$ & $\begin{array}{l}\text { Passo } \\
\text { Fundo }\end{array}$ & & & & \\
\hline \multicolumn{7}{|l|}{ Ciclo precoce } \\
\hline Cep 16-Timbó & 80abcde & $77 \mathrm{bc}$ & 55 & $62 \mathrm{bcd}$ & $90 \mathrm{abc}$ & 73 de \\
\hline FT-Saray & $82 \mathrm{abcd}$ & $80 \mathrm{abc}$ & 62 & $70 \mathrm{abc}$ & $92 \mathrm{ab}$ & 77abcd A \\
\hline IAS 5 & 73 bcde & $75 \mathrm{bc}$ & 57 & $55 \mathrm{~d}$ & $76 \mathrm{c}$ & $67 \mathrm{~g} \mathrm{~A}$ \\
\hline Ivorá & $65 \mathrm{e}$ & \multirow{2}{*}{$\begin{array}{l}75 \mathrm{bc} \\
87 \mathrm{ab}\end{array}$} & 53 & $65 \mathrm{abcd}$ & $88 \mathrm{abc}$ & fg $\mathrm{A}$ \\
\hline Ocepar 14 & $88 \mathrm{abc}$ & & 63 & $70 \mathrm{abc}$ & $89 \mathrm{abc}$ & $79 \mathrm{abc} \quad \mathrm{A}$ \\
\hline Média $^{2}$ & 78 & 79 & 58 & 64 & $87 \quad$ B & $74 \quad$ B \\
\hline \multicolumn{7}{|l|}{ Ciclo médio } \\
\hline Bragg & 79abcde & $73 \mathrm{bc}$ & 57 & 62 bcd & $77 \mathrm{bc}$ & 70 efg $B$ \\
\hline BR-4 & $93 \mathrm{a}$ & $87 \mathrm{ab}$ & 67 & $70 \mathrm{abc}$ & $97 \mathrm{a}$ & $83 a \quad A$ \\
\hline BR-16 & $90 \mathrm{ab}$ & $82 a b c$ & 55 & $63 \mathrm{bcd}$ & $91 \mathrm{abc}$ & $76 \mathrm{bcd}$ \\
\hline Cep 12-Cambará & 80 abcde & $75 \mathrm{bc}$ & 58 & $67 \mathrm{abcd}$ & $81 a b c$ & 72 def $B$ \\
\hline Davis & $83 a b c d$ & $77 \mathrm{bc}$ & 60 & $58 \mathrm{~cd}$ & $87 a b c$ & $73 \mathrm{de}$ \\
\hline IAS 4 & $83 \mathrm{abcd}$ & $82 \mathrm{abc}$ & 62 & $65 \mathrm{abcd}$ & $89 a b c$ & 76 bcd \\
\hline Ipagro 21 & 70 de & $81 \mathrm{abc}$ & 68 & $73 \mathrm{ab}$ & $94 \mathrm{a}$ & 75 cde $A B$ \\
\hline RS 7-Jacuí & 75 bcde & $72 \mathrm{c}$ & 60 & $55 \quad \mathrm{~d}$ & $91 \mathrm{abc}$ & $71 \quad \operatorname{defg} \mathrm{A}$ \\
\hline Média $^{2}$ & $82 \quad \mathrm{AB}$ & $78 \quad \mathrm{~B}$ & 60 & $64 \quad$ B & $88 \quad \mathrm{~B}$ & 75 \\
\hline Ciclos semitardio e tardio & & & & & & \\
\hline Cep 20-Guajuvira & 80abcde & $83 a b c$ & 58 & $68 \mathrm{abc}$ & $96 a$ & 77abcd $\mathrm{AB}$ \\
\hline FT-Abyara & $83 \mathrm{abcd}$ & $70 \mathrm{c}$ & 50 & $58 \mathrm{~cd}$ & $77 \mathrm{bc}$ & $\begin{array}{lll}68 & \mathrm{~g} & \mathrm{~B}\end{array}$ \\
\hline RS 9-Itaúba & 70 de & $92 \mathrm{a}$ & 68 & $73 a b$ & $93 \mathrm{a}$ & $79 a b c \quad A$ \\
\hline Cobb & $90 \mathrm{ab}$ & $87 \mathrm{ab}$ & 63 & $70 \mathrm{abc}$ & $97 \mathrm{a}$ & $81 \mathrm{ab}$ \\
\hline RS 5-Esmeralda & $92 \mathrm{a}$ & $87 \mathrm{ab}$ & 62 & $77 \mathrm{a}$ & $96 a$ & $83 a$ \\
\hline Média $^{2}$ & 83 & $85 \mathrm{~A}$ & 60 & 69 & 92 & 78 \\
\hline Média geral & 81 & 80 & 59 & 66 & 89 & 75 \\
\hline $\mathrm{CV}(\%)$ & 6,61 & 5,83 & 13,33 & 5,96 & 5,84 & - \\
\hline F cultivares/ambientes & $* *$ & $* *$ & ns & $* *$ & $* *$ & $* *$ \\
\hline F entre médias de ciclos & $*$ & $* *$ & $\mathrm{~ns}$ & $* *$ & $*$ & $* *$ \\
\hline
\end{tabular}

${ }^{1}$ Médias não seguidas pela mesma letra minúscula, nas colunas, indicam que as cultivares dentro de cada ambiente e na média geral, independentemente de ciclo, diferem segundo o teste de Tukey, em nível de $5 \%$ de probabilidade de erro.

${ }^{2}$ As médias de cada ciclo, no mesmo ambiente, e das cultivares, na última coluna, não seguidas pela mesma letra maiúscula, diferem segundo o teste de Tukey, em nível de $5 \%$ de probabilidade de erro.

inferiores a $3.500 \mathrm{~kg} / \mathrm{ha}$, para a maior parte das cultivares avaliadas nestes ensaios.

Os resultados aqui discutidos evidenciam que, em semeaduras realizadas durante os dois últimos decêndios de dezembro, podem-se utilizar, no Planalto Médio do Rio Grande do Sul, cultivares de soja de ciclos precoce e médio, sem comprometer a produtividade de grãos, em comparação com as de ciclo tardio. Com isso, a área poderia ser desocupada mais cedo, facilitando a implantação de sistemas de rotação de culturas, reduzindo o tempo de ocorrência de pragas, especialmente de percevejos, e permitindo que a colheita fosse feita durante um período no qual, normalmente, ocorrem condições climáticas mais favoráveis, evitando-se que a mesma coincida com períodos de excesso de precipitação pluvial no final do ciclo.

\section{CONCLUSÕES}

As cultivares de soja atualmente indicadas para cultivo no Rio Grande do Sul apresentam 
Tabela 4 - Resultados médios de altura de inserção dos legumes inferiores, em cm, obtidos nos experimentos de cultivares de soja de diferentes ciclos em Cruz Alta (RS) nas safras agrícolas de 1994/95 a 1996/97 e em Passo Fundo (RS) nas safras de 1994/95 e de 1997/98. Passo Fundo (RS), 2000.

\begin{tabular}{|c|c|c|c|c|c|c|c|}
\hline \multirow{3}{*}{ Ciclo/Cultivar } & \multicolumn{6}{|c|}{ Ambiente $^{1}$} & \multirow{3}{*}{$\begin{array}{c}\text { Média }^{1 \text { e } 2} \\
\text { Geral }\end{array}$} \\
\hline & \multicolumn{2}{|c|}{$1994 / 95$} & \multirow{2}{*}{\multicolumn{2}{|c|}{$\begin{array}{c}\text { 1995/96 } \\
\text { Cruz } \\
\text { Alta }\end{array}$}} & \multirow{2}{*}{$\begin{array}{c}\text { 1996/97 } \\
\text { Cruz } \\
\text { Alta }\end{array}$} & \multirow{2}{*}{$\begin{array}{l}\text { 1997/98 } \\
\text { Passo } \\
\text { Fundo }\end{array}$} & \\
\hline & $\begin{array}{l}\text { Cruz } \\
\text { Alta }\end{array}$ & $\begin{array}{l}\text { Passo } \\
\text { Fundo }\end{array}$ & & & & & \\
\hline \multicolumn{8}{|l|}{ Ciclo precoce } \\
\hline Cep 16-Timbó & $17 \mathrm{ab}$ & 14 & $8 \mathrm{c}$ & & $13 \mathrm{~cd}$ & 16 & 14 \\
\hline FT-Saray & $16 a b$ & 14 & $11 \mathrm{abc}$ & & $14 \mathrm{bcd}$ & 18 & 15 \\
\hline IAS 5 & $17 \mathrm{ab}$ & 16 & $10 \mathrm{abc}$ & & $13 \mathrm{~cd}$ & 17 & 15 \\
\hline Ivorá & $14 \mathrm{~b}$ & 15 & $9 \mathrm{c}$ & & $14 \mathrm{bcd}$ & 21 & 15 \\
\hline Ocepar 14 & $17 \mathrm{ab}$ & 12 & $10 \mathrm{abc}$ & & 14 bcd & 15 & 14 \\
\hline Média $^{2}$ & 16 & 14 & 10 & B & 14 B & 17 & 15 \\
\hline \multicolumn{8}{|l|}{ Ciclo médio } \\
\hline Bragg & $16 \mathrm{ab}$ & 17 & $10 \mathrm{abc}$ & & $15 \mathrm{bcd}$ & 21 & 16 \\
\hline BR-4 & $17 \mathrm{ab}$ & 15 & $11 \mathrm{abc}$ & & $14 \mathrm{bcd}$ & 16 & 15 \\
\hline BR-16 & $18 \mathrm{a}$ & 14 & $10 \mathrm{abc}$ & & $14 \mathrm{bcd}$ & 20 & 15 \\
\hline Cep 12-Cambará & $19 \mathrm{a}$ & 10 & $9 \mathrm{c}$ & & $12 \mathrm{~d}$ & 14 & 13 \\
\hline Davis & $15 \mathrm{ab}$ & 12 & $12 \mathrm{abc}$ & & $13 \mathrm{~cd}$ & 17 & 14 \\
\hline IAS 4 & $16 a b$ & 15 & $10 \mathrm{abc}$ & & $12 \mathrm{~d}$ & 17 & 14 \\
\hline Ipagro 21 & $15 \mathrm{ab}$ & 17 & $10 \mathrm{abc}$ & & 14 bcd & 16 & 14 \\
\hline RS 7-Jacuí & $16 \mathrm{ab}$ & 14 & $11 \mathrm{abc}$ & & 14 bcd & 17 & 15 \\
\hline Média $^{2}$ & 16 & 14 & 10 & $\mathrm{~B}$ & $14 \quad \mathrm{~B}$ & 17 & 15 \\
\hline \multicolumn{8}{|l|}{ Ciclos semitardio e tardio } \\
\hline Cep 20-Guajuvira & $19 \mathrm{a}$ & 14 & $12 \mathrm{abc}$ & & $18 \mathrm{ab}$ & 14 & 15 \\
\hline FT-Abyara & $15 \mathrm{ab}$ & 13 & $11 \mathrm{abc}$ & & $16 a b c$ & 17 & 14 \\
\hline RS 9-Itaúba & $15 \mathrm{ab}$ & 14 & $14 \mathrm{a}$ & & $20 \mathrm{a}$ & 14 & 15 \\
\hline Cobb & $18 \mathrm{a}$ & 16 & $11 \mathrm{abc}$ & & $15 \mathrm{bcd}$ & 21 & 16 \\
\hline RS 5-Esmeralda & $18 \mathrm{a}$ & 14 & $14 \mathrm{a}$ & & $16 \mathrm{abc}$ & 15 & 15 \\
\hline Média ${ }^{2}$ & 17 & 14 & 12 & A & $17 \quad \mathrm{~A}$ & 16 & 15 \\
\hline Média geral & 16 & 14 & 11 & & 15 & 17 & 15 \\
\hline $\mathrm{CV}(\%)$ & 9,17 & 16,26 & 13,00 & & 9,30 & 17,42 & - \\
\hline F cultivares/ambientes & $* *$ & ns & $* *$ & & $* *$ & ns & ns \\
\hline F entre médias de ciclos & ns & ns & $* *$ & & $* *$ & ns & ns \\
\hline
\end{tabular}

${ }^{1}$ Médias não seguidas pela mesma letra minúscula, nas colunas, indicam que as cultivares dentro de cada ambiente e na média geral, independentemente de ciclo, diferem segundo o teste de Tukey, em nível de $5 \%$ de probabilidade de erro.

${ }^{2}$ As médias de cada ciclo ,no mesmo ambiente, e das cultivares, na última coluna, não seguidas pela mesma letra maiúscula, diferem segundo o teste de Tukey, em nível de $5 \%$ de probabilidade de erro.

rendimento de grãos, altura de planta e inserção dos legumes inferiores semelhantes, quando semeadas no período compreendido entre 10 a 30 de dezembro na região do Planalto Médio, independentemente de seu ciclo.

\section{REFERÊNCIAS BIBLIOGRÁFICAS}

BARNI, N.A., BERGAMASCHI, H. Alguns princípios técnicos para a semeadura. In: MIYASAKA, S., MEDINA, J.C. (Eds.). A soja no Brasil. Campinas : ITAL, 1981. Cap.10, p.476-480.
BARNI, N.A., BERGAMASCHI, H., GOMES, J.E. de S. Época de semeadura e cultivares de soja para o Rio Grande do Sul. IPAGRO Informa, Porto Alegre, n.21, p.67-70, 1978.

BARNI, N.A., GOMES, J.E. de S., HILGERT, E.R., et al. Épocas de semeadura de cultivares de soja para o Rio Grande do Sul. IPAGRO Informa, Porto Alegre, n.28, p.25-30, 1985 .

BERLATO, M., WESTPHALEN, S. Resultados preliminares do ensaio ecológico de soja: Período de 1967/68-1970/71. In: REUNIÃO DA COMISSÃO TÉCNICA DE SOJA, 15., 1971, Porto Alegre, RS. Súmula dos trabalhos fitotécnicos na cultura da soja. Porto Alegre : Secretaria da Agricultura, 1971. p.87-122.

BONATO, E.R., BERTAGNOLLI, P.F., IGNACZAK, J.C., et al. Desempenho de cultivares de soja em três épocas de semeadura, no Rio Grande do Sul. Pesq Agropec Bras, v.33, n.6, p.879-884, 1998.

COCKRAN, W.G. The combination of Estimates from different experiments. Biometrics, v.10, p.101-129, 1954.

LÚCIO, A.D., STORCK, L., BANZATTO, D.A. Classificação dos experimentos de competição de Cultivares quanto a sua precisão. Pesq Agrop Gaúcha, v.5, n.1, p.99-103, 1999.

MOTA, F.S. da, GARCEZ, J.R.B., BONATO, E.R., et al. Época de semeadura da soja no Rio Grande do Sul e Santa Catarina. A Granja, v.29, n.310, p.32-33, 1973.

REUNIÃO DE PESQUISA DE SOJA DA REGIÃO SUL, 22, 1994, Cruz Alta, RS. Recomendações técnicas para a cultura da soja no Rio Grande do Sul e Santa Catarina 1994/95. Cruz Alta : FUNDACEP/FECOTRIGO, 1994. 66p.

REUNIÃO DE PESQUISA DE SOJA DA REGIÃO SUL, 27, 1999, Chapecó, SC. Recomendações técnicas para a cultura da soja no Rio Grande do Sul e Santa Catarina 1999/2000. Chapecó : Epagri/CPPP, 1999. 167p.

RUBIN, S. de A.L. Progresso no melhoramento genético de soja no Estado do Rio Grande do Sul. Santa Maria, 1995. 73p. Dissertação (Mestrado em Agronomia) - Curso de Produção Vegetal, Universidade Federal de Santa Maria, 1995. 